\title{
Appointment of a new Co-EIC, Dr. Michael Maskos
}

\section{Microsystem technologies, March 2021}

\author{
Bharat Bhushan ${ }^{1}$
}

Published online: 27 April 2021

(c) The Author(s), under exclusive licence to Springer-Verlag GmbH Germany, part of Springer Nature 2021

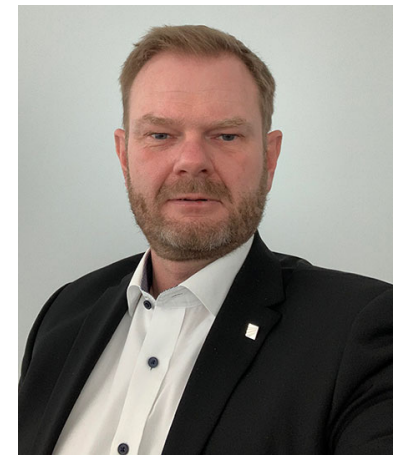

Microsystem technologies (MST)-micro- and nanosystems, and information storage and processing systems - is a premier archival journal with high impact factor. It provides rapid publication of important and timely results on electromechanical, materials science, design, and manufacturing issues of these systems and their components. The micro- and nanosystems include MEMS/ NEMS (micro/nanoelectromechanical systems), e.g., sensors and actuators, micromechatronics, optoelectronics, microfluidics and systems integration. Information storage systems include magnetic recording, optical recording, and other recording devices, processing systems include copiers, printers, scanners and digital cameras.

MST was launched in 1994 by Dr. Herbert Rauch of Fraunhofer Institute for Reliability and Microintegration (IZM), Berlin. Dr. Bharat Bhushan of The Ohio State University became Co-Editor-in-Chief (Co-EIC) in 2002. Dr. Bernd Michel of IZM replaced Dr. Rauch in Jan 2007 and retired in Dec. 2020.

Bharat Bhushan

Bhushan.2@osu.edu

https://www.springer.com/journal/542

1 The Ohio State University, Columbus, Ohio, USA
It is my distinct pleasure to announce the appointment, of Dr. Michael Maskos as Co-EIC, who is Executive Director of Fraunhofer Institute for Microengineering and Microsystems (IMM), Mainz, Germany, since 2011. He holds a full professorship in the field of Chemical Process Engineering / Microfluidics at the Johannes Gutenberg University Mainz, Germany. In 2000, he received the Research Award from the Boehringer-Ingelheim Foundation and in the same year went to McGill University in Montreal, Canada, as a research scholar of the German Academy of Natural Scientists Leopoldina. After returning, he served as the leader of the Division Durability of Polymers at the Federal Institute of Materials Research and Testing (BAM). In 2015, he won the Literature Prize of the Chemical Industry of Germany for the textbook "Polymers: Synthesis, Characteristics and Applications".

His expertise includes optical, mechanical, and clean room-based processes for microstructuring, the application-oriented focus related to microfluidics-based diagnostics and analytics, and nanomaterials for various applications. His personal goal is to secure the journal's long-term success with new ideas with increasing impact factor. Pl join me in welcoming Dr. Maskos to the MST editorial board.

Dr. Bharat Bhushan, Co-EIC

Academy Professor, The Ohio State University

March 2021

Publisher's Note Springer Nature remains neutral with regard to jurisdictional claims in published maps and institutional affiliations. 